\section{Mortalidade neonatal precoce hospitalar em Minas Gerais: associação com variáveis assistenciais e a questão da subnotificação*}

\section{Early neonatal hospital mortality in Minas Gerais: association with healthcare variables and the issue of underreporting}

\author{
Deise Campos ${ }^{1}$ \\ Rosangela Helena Loschi ${ }^{2}$ \\ Elisabeth França ${ }^{3}$ \\ ${ }^{1}$ Faculdade de Medicina, Universidade Federal de Minas Gerais \\ ${ }^{2}$ Departamento de Estatística, Instituto de Ciências Exatas, Universidade Federal \\ de Minas Gerais \\ ${ }^{3}$ Departamento de Medicina Preventiva e Social, Faculdade de Medicina, \\ Universidade Federal de Minas Gerais
}

*Trabalho baseado em dissertação de mestrado apresentada ao Programa de Pós-Graduação em Saúde Pública da Universidade Federal de Minas Gerais em 2006.

Apoio financeiro: Este trabalho foi parcialmente financiado pela FAPEMIG (Processo EDT 1770/ 2003) e CNPq (Processo $n^{\circ}$. 403707/04-8). RHL tem sua pesquisa parcialmente financiada pelo CNPq (Processos no. 3004505/2006-4; 472877/2006-2; 472066/2004-8). DC recebeu bolsa de mestrado da CAPES.

Correspondência: Elisabeth Barboza França. Av. Alfredo Balena 190, $9^{\circ}$ andar - Belo Horizonte, Minas Gerais CEP 30130-100. E-mail: efranca@medicina.ufmg.br

\section{Resumo}

Introdução: Os óbitos neonatais precoces estão relacionados com problemas de acesso à assistência de qualidade ao pré-natal, ao parto hospitalar e ao recém-nascido. Os hospitais em Minas Gerais estão distribuídos de forma heterogênea e isto pode se refletir em diferentes níveis de mortalidade neonatal $(\mathrm{MN})$ entre as regiões do Estado. Objetivo: Investigar a MN precoce hospitalar no Sistema de Informações Hospitalares (SIH/SUS) e avaliar possível associação da taxa de mortalidade neonatal precoce obtida a partir do SIH/SUS (TMNP_SIH), com variáveis relativas ao atendimento à gestante e ao recém-nascido em estratos de municípios homogêneos. Método: Utilizou-se o SIH/SUS para obter o número de nascimentos e óbitos segundo o município de residência. Os municípios foram agrupados segundo microrregião e tamanho populacional, totalizando 199 grupamentos. O método CART (Classification and Regression Tree) identificou três estratos de grupamentos de municípios homogêneos do ponto de vista socioeconômico. Para cada estrato utilizouse a matriz de correlação de Spearman para avaliar associação entre a TMNP e indicadores da assistência. Resultados: A TMNP_SIH para Minas Gerais foi de 10,9/ 1000 nascidos vivos. Observou-se maior probabilidade de TMNP_SIH menor que 8/ 1000 nascidos vivos onde a situação socioeconômica é mais precária. Observou-se correlação positiva entre TMNP_SIH e berços por mulher em idade fértil e baixo peso ao nascer no Estrato 1, e entre TMNP_SIH e médicos por habitante no Estrato 3. Conclusão: Questões relativas ao acesso à assistência, sub-registro de óbitos e erro de classificação de neomorto como natimorto podem estar se refletindo na TMNP_SIH. O volume de nascimentos e óbitos registrados no SIH/SUS justifica investimentos na qualidade desses registros e sua utilização em estudos epidemiológicos.

Palavras-chave: Mortalidade neonatal. Fatores socioeconômicos. Sistemas de informação. Registros de mortalidade. Subregistro. SUS (BR). 
Abstract

Introduction: Early neonatal deaths are related to access to good quality prenatal, hospital delivery and newborn health care. In Minas Gerais, Brazil, hospitals are heterogeneously distributed, which may result in various levels of neonatal mortality among the State's regions. Objective: To investigate early neonatal hospital mortality in the Hospital Information System (SIH/SUS) and assess possible associations between early neonatal hospital mortality rates (ENMR_SIH) and variables regarding care to pregnant women and the newborn in strata of homogeneous cities. Method: The SIH/SUS was used to obtain the number of births and deaths according to place of residence. Cities were grouped according to micro-region and population size, totaling 199 groups. The CART (Classification and Regression Tree) identified three strata of homogeneous city groups according to socioeconomic factors. For each stratus, a Spearman correlation matrix was used to evaluate associations between rates and health care indicators. Results: The ENMR_SIH for Minas Gerais was 10.9/ 1000 live births. A greater probability of an ENMR_SIH under 8/1000 live births was observed in areas with poorer socioeconomic conditions. A positive correlation between the ENMR_SIH and cribs per woman in fertile age and the proportion of low birth weight in Strata 1, and between the ENMR_SIH and physicians per inhabitant in Strata 3 was observed. Conclusion: Issues concerning access to healthcare, underreporting of deaths and the misclassification of live births as stillbirths may be reflected in rates. The number of births and deaths registered in the SIH/ SUS justifies studies on the data collected and investments in the quality of those records.

Keywords: Neonatal mortality. Socioeconomic factors. Information systems. Death registration. Underreporting. SUS (BR).

\section{Introdução}

No Brasil, nas últimas décadas, a mortalidade neonatal precoce vem aumentando sua participação relativa na mortalidade infantilil. Embora esta seja uma tendência esperada, conforme observado nos países desenvolvidos ${ }^{2}$, persistem questões relativas aos elevados níveis e seus determinantes que devem ser abordados.

A desigualdade social e econômica pode ser vista como o principal determinante no aumento do risco de morte dos neonatos, uma vez que tal desigualdade está associada a problemas de saúde materna e a dificuldades no acesso a cuidados médicos neonatais. Diferenciais nos níveis de escolaridade da mãe e nos níveis de renda estão freqüentemente associados a diferenciais nas taxas de mortalidade infantil ${ }^{3,4}$. Algumas variáveis, como idade materna, escolaridade e ocupação podem afetar a percepção da paciente quanto aos seus próprios problemas de saúde e a utilização dos serviços de saúde. Por outro lado, os fatores socioeconômicos interferem na oferta de serviços e no acesso a assistência de qualidade ${ }^{5}$.

Variáveis relativas à assistência, como o número de médicos por habitante e de consultas no pré-natal, podem demonstrar a oportunidade da oferta e do acesso a serviços de atenção à gestante, ao parto e ao recém-nascido ${ }^{6}$. A reduzida oferta de serviços, como por exemplo a ausência de atendimento pediátrico na sala de parto, pode estar associada à maior mortalidade neonatal precoce e, como conseqüência, a magnitude das taxas da mortalidade indicaria a qualidade dos serviços presta$\operatorname{dos}^{7}$.

Em Minas Gerais, a taxa de mortalidade neonatal precoce (TMNP) pode ser considerada muito elevada se comparada à observada em outros Estados das regiões Sudeste e Sul e em países mais desenvolvidos $^{1,8}$. O seu monitoramento torna-se então uma questão fundamental, a ser feito prioritariamente nos hospitais, que é onde ocorre a maior parte desses óbitos? 
Nota-se que, apesar de Minas Gerais concentrar $10 \%$ dos estabelecimentos que atendem ao parto no país ${ }^{9}$, o equipamento hospitalar distribui-se de forma heterogênea, com grande concentração de estabelecimentos de grande porte na macrorregião Centro, onde se localiza a Região Metropolitana de Belo Horizonte ${ }^{10}$.

Semelhantemente, a qualidade das informações produzidas no Estado não se apresenta de forma homogênea. Minas Gerais é o único Estado da Região Sudeste cujas taxas de mortalidade infantil (TMI) oficiais são estimadas pelo Instituto Brasileiro de Geografia e Estatística (IBGE) a partir de métodos indiretos, devido ao subregistro de nascimentos no Sistema de Informações sobre Nascidos Vivos (SINASC) ${ }^{11}$. A Fundação João Pinheiro $(2005)^{\mathrm{a}}$, avaliando as microrregiões do Estado, encontrou que o grau de cobertura de nascimentos pelo SINASC alcança níveis muito baixos, como, por exemplo, na microrregião Minas Novas/Turmalinas, com somente $50 \%$ de cobertura. Os óbitos infantis registrados no SIM atingem somente $16 \%$ de cobertura na microrregião de Araçuaí.

Face aos problemas de sub-registro de óbitos e nascimentos no SIM (Sistema de Informações sobre Mortalidade) e SINASC, Schramm e Szwarcwald (2000b) ${ }^{12}$ utilizaram o Sistema de Informações Hospitalares do Sistema Único de Saúde (SIH/SUS) e verificaram que este sistema captou melhor que o SIM os óbitos no período neonatal precoce nas regiões Norte e Nordeste do país.

Considerando a ampla cobertura ao parto dada pela rede hospitalar do SUS, especialmente no interior do Estado de Minas Gerais ${ }^{9}$, e que os óbitos neonatais precoces são predominantemente hospitalares $^{7}$, propõe-se investigar a mortalidade neonatal precoce hospitalar em municípios mineiros utilizando o SIH/SUS e avaliar uma possível associação entre a taxa de mortalidade neonatal precoce hospitalar com variáveis relativas ao atendimento à gestante e ao recém-nascido em estratos de municípios homogêneos do ponto de vista socioeconômico.

\section{Métodos}

\section{Delineamento do estudo}

Trata-se de estudo ecológico em que foram estudados todos os nascidos vivos hospitalares de Minas Gerais nos anos de 1999, 2000 e 2001 registrados no SIH/SUS, e os que faleceram antes de completar sete dias de vida (óbito neonatal precoce hospitalar). Considerou-se o coeficiente médio trienal da mortalidade neonatal precoce hospitalar para se obter maior estabilidade das taxas.

Atualmente, o Estado de Minas Gerais, com seus 853 municípios, está dividido em 13 macrorregiões e 75 microrregiões ${ }^{13}$. Os nascimentos e óbitos ocorridos nos hospitais foram somados segundo o município de residência da mãe ou do recém nascido (município de procedência do paciente). Os hospitais foram agrupados segundo classificação de tamanho populacional dos municípios, respeitando os limites das microrregiões do Estado. Foram definidos os seguintes grupos de municípios:

- municípios de até 19.999 habitantes;

- de 20.000 a 49.999 habitantes;

- de 50.000 a 99.999 habitantes;

- de 100.000 habitantes ou mais; e

- municípios-pólo de microrregiões.

Considerou-se este último grupo independentemente do tamanho da população do município, por causa de suas características de referência microrregional. No caso de microrregião com mais de um município-pólo, os mesmos foram reunidos.

Cento e noventa e nove grupamentos foram obtidos e são as unidades de análi-

a Fundação João Pinheiro. Centro de Estudos Econômicos e Sociais. Mensuração e acompanhamento do nível da mortalidade infantil de Minas Gerais. - Belo Horizonte, 2005. 99 p.il. 
se deste estudo. Este procedimento teve dois objetivos:

- evitar instabilidade estatística associada ao uso de pequenos números, obtendo-se pelo menos 250 nascidos vivos (NV) em cada grupamento; e

- estratificar os municípios segundo o tamanho populacional, que é uma variável que pode estar refletindo diferentes graus de urbanização ${ }^{6}$.

As populações dos municípios foram obtidas do Censo Demográfico de $2000^{14}$.

\section{Coleta de dados no SIH/SUS}

A metodologia utilizada para obtenção de dados do SIH/SUS foi adaptada do método descrito por Schramm \& Szwarcwald $(2000 b)^{12}$. Para obtenção do número de nascidos vivos a partir dos arquivos no CDRom do Ministério da Saúde, foram selecionadas as Autorizações de Internação Hospitalar (AIHs) emitidas para mulheres internadas no período de 01/01/1999 a 31/ 12/2001 cujo campo “nascido_vivo” estivesse preenchido. Esta variável refere-se ao neonato e só é preenchida em caso de parto. Em seguida, foram extraídas as AIHs cujo campo "saída_óbito" estivesse preenchido, pois se refere ao óbito do neonato. Foram selecionadas as AIHs emitidas para crianças menores de sete dias nascidas entre 01/01/1999 e 31/12/2001, com o campo "cobrança” preenchido como óbito. Nos dois bancos de dados criados, foram consideradas as informações sobre mortalidade neonatal precoce até 07/01/2002. Estimou-se a idade da criança que foi a óbito no período neonatal precoce de duas formas:

- nas AIHs das mães: data de saída menos data de internação; e

- nas AIHs próprias das crianças: data de saída menos data de nascimento.

Após estes procedimentos, criou-se um único banco de dados das AIHs.

\section{Variáveis selecionadas}

Considerou-se como variável depen- dente a taxa de mortalidade neonatal precoce hospitalar do SUS (TMNP_SIH) que foi obtida dividindo-se o número de óbitos em menores de sete dias pelo número de NV registrados nas AIHs das mães e nas AIHs dos recém-nascidos (por mil NV). A TMNP_SIH foi transformada em variável dicotômica, tendo como ponto de corte o valor obtido para o município de Belo Horizonte, que foi de aproximadamente 8/ $1000 \mathrm{NV}$, e então utilizada como variável resposta no método de regressão escolhido. Utilizou-se este valor, pois considerouse que a capital do Estado tem informação de melhor qualidade e rede de assistência melhor qualificada para o atendimento ao parto e ao recém-nascido.

Como variáveis independentes, consideraram-se variáveis socioeconômicas e assistenciais. As variáveis socioeconômicas para os grupamentos selecionados foram obtidas pelo cálculo das médias ponderadas das proporções ao nível municipal coletadas do Atlas do Desenvolvimento Humano de $2003^{14}$. Essas variáveis são as seguintes:

Variáveis relativas à renda: Desigualdade de renda: razão da renda média dos $10 \%$ mais ricos e dos $40 \%$ mais pobres. Pobreza: percentual de pessoas com renda per capita abaixo de meio salário mínimo.

Variáveis relativas à escolaridade: Alta escolaridade: percentual de pessoas de 25 anos ou mais de idade com doze anos ou mais de estudo. Analfabetismo funcional: percentual de pessoas de 25 anos ou mais com menos de quatro anos de estudo. Analfabetismo: percentual de pessoas de 15 anos ou mais analfabetas.

Variáveis relativas a características dos domicílios: Água encanada: percentual de pessoas que vivem em domicílios com água encanada. Domicílio subnormal: percentual de pessoas que vivem em domicílios subnormais (favelas ou assemelhados, em sua maioria carentes de serviços públicos essenciais). Densidade por dormitório: percentual de pessoas que vivem em domicílios com densidade acima de duas pessoas por dormitório. 
Os dados relativos à assistência foram obtidos de três fontes diferentes: no CDRom SIM-SINASC do Ministério da Saúde, da Pesquisa Assistência Médico-Sanitária de 1999 (PAMS-99) e da Rede Hospitalar SUS disponíveis no site do DATASUS ${ }^{8}$. Não foram incluídos os dados de registros como de "município ignorado". Foram consideradas as seguintes variáveis assistenciais:

- Médicos por habitantes: número de médicos por mil habitantes;

- Leitos por habitante: número mensal médio de leitos da rede SUS por mil habitantes;

- Berços por mulher: número de berços por mil mulheres em idade fértil;

- Sete ou mais consultas no pré-natal: proporção de crianças nascidas vivas cujas mães realizaram sete ou mais consultas no pré-natal;

- Baixo peso ao nascer: proporção de crianças nascidas vivas com peso ao nascer menor que 2.500 gramas;

- Causas mal definidas: proporção de óbitos em crianças menores de um ano sem definição da causa básica; e

- Cobertura do PSF: proporção de pessoas cadastradas no programa de saúde da família do município.

\section{Análise estatística}

A análise foi realizada em três etapas. Inicialmente, para identificar os fatores socioeconômicos mais fortemente relacionados com a taxa de mortalidade neonatal precoce hospitalar (dicotomizada em $\geq 8 / 1000 \mathrm{NV}$ e $<8 / 1000 \mathrm{NV}$ ) e identificar os grupamentos de municípios mais homogêneos segundo esses fatores, utilizou-se o método "CART" - Classification and Regression Tree, que pode ser considerado como um modelo de regressão nãoparamétrico e é robusto com respeito a valores atípicos. O modelo CART é representado por uma árvore de classificação, onde cada nó corresponde à informação proveniente de uma variável especificada. Pelo método, o conjunto original de dados é dividido em subconjuntos cada vez mais homogêneos mediante divisões binárias. O processo de divisão considera todas as combinações possíveis das variáveis independentes, e as interações entre as variáveis são tratadas automaticamente. O processo é repetido até que alguma das regras de divisão aplicadas seja atingida, originando nós terminais os mais homogêneos possíveis. Quando a variável dependente é dicotômica, em cada nó terminal obtém-se a probabilidade condicional de ocorrência de sucesso, caso todas as características apontadas no ramo ao qual pertence o nó estiverem satisfeitas ${ }^{15,16}$.

As seguintes regras de parada de divisão foram utilizadas na análise: índice Gini como medida de impureza para as divisões dos subconjuntos de dados, com nível mínimo de melhora na impureza menor que 0,0001 (Improvement), número máximo de camadas fixado em 10 níveis, mínimo de casos no nó-pai fixado em dez e no nó-filho em cinco casos (por ser este o número máximo de grupamentos de municípios em cada microrregião). Foi selecionado o comando Grow tree and Prune, que fornece o modelo (árvore) mais parcimonioso dentre todas as árvores mais adequadas para descrever o comportamento do conjunto de dados analisados.

Para avaliar a qualidade do modelo, foi utilizada a Matriz de risco de classificação fornecida pelo CART, que informa a porcentagem de classificação incorreta feita pelo modelo. Avaliou-se a adequação do modelo pela curva ROC ou Receiver Operating Characteristic, indicada para avaliar se o modelo obtido pelo CART classificava corretamente um grupamento de municípios como tendo TMNP_SIH menor que 8 por mil NV ou maior ou igual a 8 por mil NV. A curva ROC é um gráfico que relaciona a sensibilidade com o complemento da especificidade e a área sob a curva resultante expressa o desempenho do modelo ${ }^{17}$. O modelo ideal deve ter valores distantes de 0,5 e próximos de 1,0 para a área sob a curva ROC e próximos de zero para o risco de classificação do CART. Para 
avaliar se as médias das variáveis diferem entre os estratos, utilizamos o teste de Kruskal-Wallis, seguido do teste de Dunn para comparações múltiplas.

Na segunda etapa da análise, foram descritas e comparadas as características assistenciais dos grupamentos de municípios em cada estrato socioeconômico fornecido pelo CART. Na terceira etapa utilizou-se o teste de correlação de Spearman, considerando nível de significância de 5\% para avaliar a associação entre a taxa de mortalidade neonatal precoce hospitalar e as variáveis assistenciais para cada um dos estratos de grupamentos de municípios.

Foram utilizados para análise os softwares Excel versão 11.0, SPSS versão 12, SigmaStat versão 3.5 e Answer Tree versão $3.0^{18}$.

\section{Resultados}

Em média, 252.112 crianças nascidas vivas e 2.745 óbitos neonatais precoces foram registrados anualmente no SIH/SUS em Minas Gerais entre 1999 e 2001. Obte- ve-se uma TMNP_SIH de 10,9/1000 NV para o Estado neste período.

A Tabela 1 apresenta a distribuição dos grupamentos de municípios segundo tamanho populacional. Somente 13 grupamentos têm menos de 20.000 habitantes, ou seja, em $93,5 \%(n=186)$ grupamentos a população estimada foi maior que 20.000 habitantes. Neste mesmo período, de 1999 a 2001, o SIH/SUS registrou 84,3\% do número total de nascimentos hospitalares do SINASC e 85,0\% do número total de óbitos neonatais precoces hospitalares registrados no SIM. Esta proporção foi diferenciada: foi maior nas macrorregiões Jequitinhonha, Nordeste, Noroeste e Região Norte de Minas, onde se observa menor nível socioeconômico. Também foi diferente em relação à população do grupamento de municípios: quanto menor o grupamento populacional, maior a razão óbitos $\mathrm{SIH}$ sobre óbitos SIM e nascimentos SIH em relação a nascimentos SINASC (dados não apresentados). Ainda no mesmo período, segundo dados do SINASC, 99,1\% dos nascimentos do Estado ocorreram em hospi-

Tabela 1 - Distribuição dos grupamentos de municípios segundo tamanho populacional, Minas Gerais, 1999-2001.

Table 1 - Municipal group distribution by populational size,Minas Gerais, 1999-2001.

\begin{tabular}{|c|c|c|c|c|}
\hline \multirow[t]{2}{*}{$\begin{array}{l}\text { Tamanho de município } \\
\text { (habitantes) }\end{array}$} & \multirow[t]{2}{*}{$\begin{array}{l}\text { Número de } \\
\text { municípios }\end{array}$} & \multirow[t]{2}{*}{$\begin{array}{l}\text { Tamanho do grupamento } \\
\text { de município (habitantes) }\end{array}$} & \multicolumn{2}{|c|}{$\begin{array}{l}\text { Grupamentos } \\
\text { de municípios }\end{array}$} \\
\hline & & & $\mathrm{n}$ & $\%$ \\
\hline \multirow[t]{5}{*}{ Menor que 20.000} & 677 & menor que 20.000 & 8 & 4,0 \\
\hline & & 20.000 a 49.999 & 23 & 11,6 \\
\hline & & 50.000 a 99.999 & 29 & 14,6 \\
\hline & & igual ou maior a 100.000 & 14 & 7,0 \\
\hline & & Total & 74 & 37,2 \\
\hline \multirow[t]{4}{*}{ De 20.000 a 49.000} & 73 & 20.000 a 49.999 & 33 & 16,6 \\
\hline & & 50.000 a 99.999 & 8 & 4,0 \\
\hline & & igual ou maior a 100.000 & 3 & 1,5 \\
\hline & & Total & 44 & 22,1 \\
\hline De 50.000 a 99.999 & 4 & 50.000 a 99.999 hab & 4 & 2,0 \\
\hline Igual ou maior a 100.000 & 4 & igual ou maior a 100.000 & 2 & 1,0 \\
\hline \multirow[t]{5}{*}{ Pólos de microrregião } & 95 & menor que 20.000 & 5 & 2,5 \\
\hline & & 20.000 a 49.999 hab. & 18 & 9,0 \\
\hline & & 50.000 a 99.999 hab & 30 & 15,1 \\
\hline & & igual ou maior a 100.000 & 22 & 11,1 \\
\hline & & Total & 75 & 37,7 \\
\hline Total & 853 & & 199 & 100,0 \\
\hline
\end{tabular}


tais. Verificou-se não haver médicos em somente um dos grupamentos de municípios e em 12 deles não havia leitos de hospitais credenciados junto ao SUS.

Árvore de classificação dos grupamentos de municípios segundo nível socioeconômico

Após a categorização, a TMNP_SIH foi maior ou igual a 8 por mil NV em $72,4 \%$ grupamentos $(\mathrm{n}=144)$. Considerando apenas as variáveis socioeconômicas para a modelagem, a desigualdade de renda foi a variável identificada pelo CART como sendo a mais fortemente associada com a TMNP_SIH dicotomizada. Posteriormen- te, foi identificada a alta escolaridade (Figura 1). O Estrato 1 (primeiro nó da árvore) ficou então constituído por 19 grupamentos, o Estrato 2 por 13 e o Estrato 3 por 167 grupamentos de municípios. A primeira divisão propiciou uma melhora do nível de impureza de $4,48 \%$, e a segunda divisão, de 2,12\%. O risco de classificação foi de 0,216 , significando que $78,4 \%$ dos grupamentos foram corretamente classificados segundo o método CART. O valor encontrado para a área sob a curva ROC foi de 0,667 (IC a 95\%: 0,575-0,759; $\mathrm{p}<0,000$ ). Dessa forma, a área sob a curva foi significantemente diferente de 0,5 e o modelo obtido pode ser considerado razoável.

Taxa de mortalidade neonatal precoce no SIH/SUS

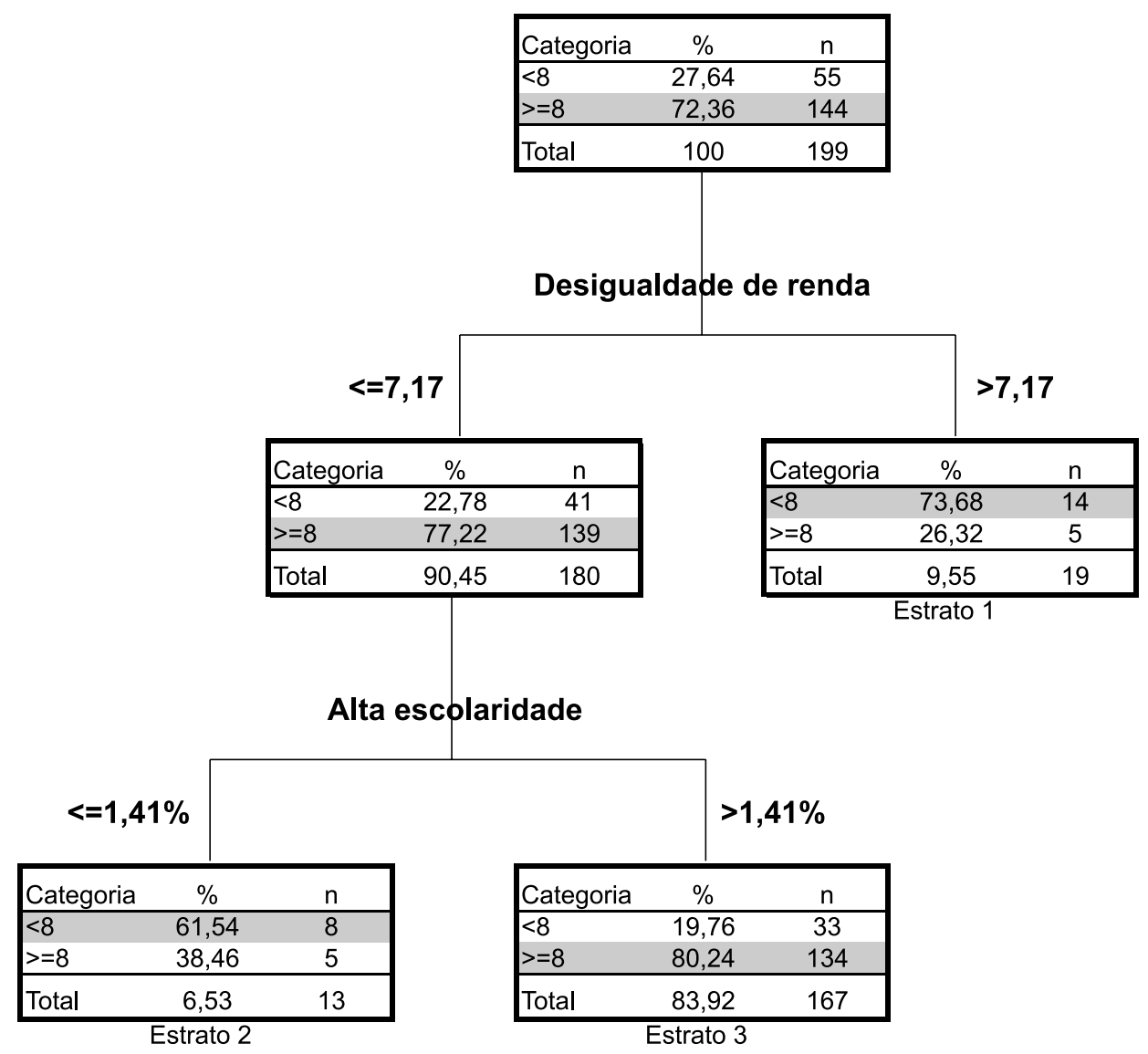

Figura 1 - Árvore de classificação dos grupamentos de municípios segundo variáveis socioeconômicas selecionadas, Minas Gerais, 1999-2001.

Figure 1 - Classification tree of city groups according to selected socioeconomic variables, Minas Gerais, 1999-2001. 
Construída a árvore de classificação, tornou-se possível obter a probabilidade de ocorrência de uma TMNP_SIH menor que 8 por mil NV condicional às variáveis explicativas selecionadas. Para o Estrato 1 esta probabilidade será de $73,68 \%$ se um grupamento de municípios apresentar uma desigualdade de renda maior que 7,17; no Estrato 2, a TMNP_SIH será menor que 8 por mil NV, com uma probabilidade de $61,54 \%$ se o grupamento de municípios apresentar proporção de pessoas com alta escolaridade menor ou igual a $1,41 \%$ e uma desigualdade de renda menor ou igual a 7,17. No Estrato 3, a probabilidade será de 19,76\% para os grupamentos de municípios com 1,41\% da população com alta escolaridade e uma desigualdade de renda menor ou igual a 7,17.

Análise descritiva dos estratos socioeconômicos de grupamentos de municípios

Os grupamentos de municípios dos Estratos 1 e 2 concentram-se predominantemente nas regiões ao norte do Estado. Foram classificados no Estrato 3 todos os grupamentos de municípios das macrorregiões Centro Sul, Leste do Sul, Oeste, Sudeste, Sul, Triângulo do Norte e Triângulo do Sul e 64 dos 75 municípios-pólo de microrregião, concentrando $91 \%$ da população do Estado.

A Tabela 2 apresenta uma análise descritiva das variáveis socioeconômicas e assistenciais nos estratos identificados e os resultados dos testes de Kruskal-Wallis e Dunn. Cabe ressaltar que apenas duas variáveis não se revelaram significativamente diferentes nos 3 estratos: “domicílios subnormais" e "cobertura do PSF". O Estrato 3 apresenta a melhor situação socioeconômica, evidenciada pelos índices de desigualdade de renda, escolaridade e taxa de pobreza. Em relação às variáveis assistenciais em geral, observou-se maior variação nos índices médios do Estrato 3, porém, mesmo assim, analisando o subconjunto como um todo, a situação assistencial observada foi melhor neste estra- to. A TMNP_SIH foi mais elevada, em média, no Estrato 3.

Para melhor visualização do comportamento das variáveis assistenciais nos três estratos, foi utilizado o recurso gráfico dos boxplots (Figura 2). Esses gráficos corroboram os resultados fornecidos pelo teste de Dunn. Verifica-se que o Estrato 1 não difere muito quanto à proporção de médicos por habitantes e de morte infantis por causas mal definidas em relação ao Estrato 2 , mas tem pior desempenho quanto à variável sete ou mais consultas no pré-natal. Verificou-se piores resultados quanto a berços por mulher no Estrato 2. O Estrato 3 tende a ter as maiores proporções de médicos por habitantes, de sete ou mais consultas no pré-natal e de baixo peso ao nascer, e as menores proporções de óbitos infantis por causas mal definidas. $\mathrm{O}$ número de leitos por habitantes e cobertura do PSF são distribuídos mais equilibradamente nos três estratos, se comparados às outras variáveis em estudo.

\section{Associação entre a taxa de mortalidade neonatal precoce hospitalar e as variáveis assistenciais}

A Tabela 3 apresenta a análise de correlação entre a TMNP_SIH e as variáveis assistenciais selecionadas nos três estratos da árvore de classificação. Percebe-se, em geral, que as correlações entre as variáveis assistenciais e a TMNP_SIH são baixas, e poucas delas são significativas, ou seja, poucas delas poderiam ajudar a explicar o comportamento da TMNP_SIH. Ainda observamos resultados significativos na faixa de $5 \%$ nos seguintes casos:

- Estrato 1: Médicos por habitantes correlacionou-se positivamente com leitos por habitantes. Cobertura do PSF correlacionou-se positivamente com sete ou mais consultas no pré-natal.

- Estrato 2: Berços por mulher e leitos por habitantes correlacionaram-se positivamente. Baixo peso ao nascer apresentou correlação negativa com leitos por habitantes e berços por mulher. 
Tabela 2 - Análise comparativa dos estratos 1, 2 e 3 segundo variáveis socioeconômicas e assistenciais, Minas Gerais, 1999-2001.

Table 2 - Comparative analises of selected variable by classification tree stratus,Minas Gerais, 1999-2001.

\begin{tabular}{|c|c|c|c|c|c|c|c|c|}
\hline \multirow[t]{2}{*}{ Variáveis } & \multirow[b]{2}{*}{ Estatística } & \multicolumn{3}{|c|}{ Estratos } & \multirow[t]{2}{*}{$\begin{array}{c}\text { Teste de } \\
\text { Kruskal-Wallis }\end{array}$} & \multicolumn{3}{|c|}{ Teste de Dunn* } \\
\hline & & 1 & 2 & 3 & & 1 vs 2 & 1 vs 3 & 2 vs 3 \\
\hline $\begin{array}{l}\text { Taxa de mortalidade neonatal } \\
\text { precoce no SIH por mil nascidos vivos }\end{array}$ & $\begin{array}{l}\text { Média } \\
\text { (DP) }\end{array}$ & $\begin{array}{l}6,80 \\
(4,62)\end{array}$ & $\begin{array}{l}7,89 \\
(4,68)\end{array}$ & $\begin{array}{l}12,24 \\
(5,97)\end{array}$ & 0,0000 & & $\operatorname{sim}$ & $\operatorname{sim}$ \\
\hline Desigualdade de renda & $\begin{array}{l}\text { Média } \\
\text { (DP) }\end{array}$ & $\begin{array}{l}8,72 \\
(1,50)\end{array}$ & $\begin{array}{l}4,82 \\
(1,01)\end{array}$ & $\begin{array}{l}4,47 \\
(1,01)\end{array}$ & 0,0000 & $\operatorname{sim}$ & $\operatorname{sim}$ & - \\
\hline Alta escolaridade (\%) & $\begin{array}{l}\text { Média } \\
\text { (DP) }\end{array}$ & $\begin{array}{l}2,35 \\
(1,15)\end{array}$ & $\begin{array}{l}1,02 \\
(0,25)\end{array}$ & $\begin{array}{l}5,47 \\
(3,33)\end{array}$ & 0,0000 & - & $\operatorname{sim}$ & $\operatorname{sim}$ \\
\hline Pobreza (\%) & $\begin{array}{l}\text { Média } \\
\text { (DP) }\end{array}$ & $\begin{array}{l}61,45 \\
(12,71)\end{array}$ & $\begin{array}{l}65,54 \\
(10,59)\end{array}$ & $\begin{array}{l}32,32 \\
(15,28)\end{array}$ & 0,0000 & - & $\operatorname{sim}$ & $\operatorname{sim}$ \\
\hline Água encanada (\%) & $\begin{array}{l}\text { Média } \\
\text { (DP) }\end{array}$ & $\begin{array}{l}59,71 \\
(17,10)\end{array}$ & $\begin{array}{l}51,29 \\
(14,26)\end{array}$ & $\begin{array}{l}89,81 \\
(12,10)\end{array}$ & 0,0000 & - & $\operatorname{sim}$ & $\operatorname{sim}$ \\
\hline Densidade por dormitório (\%) & $\begin{array}{l}\text { Média } \\
\text { (DP) }\end{array}$ & $\begin{array}{l}16,10 \\
(5,11)\end{array}$ & $\begin{array}{l}16,50 \\
(4,60)\end{array}$ & $\begin{array}{l}11,63 \\
(4,15)\end{array}$ & 0,0000 & - & $\operatorname{sim}$ & $\operatorname{sim}$ \\
\hline Analfabetismo funcional (\%) & $\begin{array}{l}\text { Média } \\
\text { (DP) }\end{array}$ & $\begin{array}{l}58,69 \\
(9,80)\end{array}$ & $\begin{array}{l}65,52 \\
(11,45)\end{array}$ & $\begin{array}{l}37,66 \\
(11,91)\end{array}$ & $\begin{array}{c}0,0000 \\
-\end{array}$ & - & $\operatorname{sim}$ & $\operatorname{sim}$ \\
\hline Analfabetismo (\%) & $\begin{array}{l}\text { Média } \\
\text { (DP) }\end{array}$ & $\begin{array}{l}26,37 \\
(6,13)\end{array}$ & $\begin{array}{l}30,71 \\
(7,82)\end{array}$ & $\begin{array}{l}13,90 \\
(6,61)\end{array}$ & 0,0000 & - & $\operatorname{sim}$ & $\operatorname{sim}$ \\
\hline Domicílios subnormais (\%) & $\begin{array}{l}\text { Média } \\
\text { (DP) }\end{array}$ & $\begin{array}{l}0,25 \\
(0,92)\end{array}$ & $\begin{array}{l}1,07 \\
(3,59)\end{array}$ & $\begin{array}{l}0,72 \\
(2,39)\end{array}$ & 0,4156 & - & - & - \\
\hline Médicos por mil habitantes (n) & $\begin{array}{l}\text { Média } \\
\text { (DP) }\end{array}$ & $\begin{array}{l}0,93 \\
(0,43)\end{array}$ & $\begin{array}{l}0,70 \\
(0,30)\end{array}$ & $\begin{array}{l}1,99 \\
(1,26)\end{array}$ & $\begin{array}{c}0,0000 \\
-\end{array}$ & & $\operatorname{sim}$ & $\operatorname{sim}$ \\
\hline Leitos do SUS por mil habitantes ( $n$ ) & $\begin{array}{l}\text { Média } \\
\text { (DP) }\end{array}$ & $\begin{array}{l}2,37 \\
(1,61)\end{array}$ & $\begin{array}{l}1,09 \\
(0,88)\end{array}$ & $\begin{array}{l}2,68 \\
(1,76)\end{array}$ & 0,0006 & $\operatorname{sim}$ & - & $\operatorname{sim}$ \\
\hline $\begin{array}{l}\text { Berços por mil mulheres em } \\
\text { idade fértil ( } n \text { ) }\end{array}$ & $\begin{array}{l}\text { Média } \\
\text { (DP) }\end{array}$ & $\begin{array}{l}1,57 \\
(1,13)\end{array}$ & $\begin{array}{l}0,72 \\
(0,61)\end{array}$ & $\begin{array}{l}1,36 \\
(0,74)\end{array}$ & 0,0105 & $\operatorname{sim}$ & - & $\operatorname{sim}$ \\
\hline $\begin{array}{l}\text { Sete ou mais consultas no } \\
\text { pré-natal (\%) }\end{array}$ & $\begin{array}{l}\text { Média } \\
\text { (DP) }\end{array}$ & $\begin{array}{l}25,54 \\
(15,86)\end{array}$ & $\begin{array}{l}26,30 \\
(13,57)\end{array}$ & $\begin{array}{l}47,22 \\
(17,10)\end{array}$ & 0,0000 & - & $\operatorname{sim}$ & $\operatorname{sim}$ \\
\hline Causas mal definidas (\%) & $\begin{array}{l}\text { Média } \\
\text { (DP) }\end{array}$ & $\begin{array}{l}19,66 \\
(12,62)\end{array}$ & $\begin{array}{l}20,55 \\
(12,37)\end{array}$ & $\begin{array}{l}12,06 \\
(9,58)\end{array}$ & 0,0017 & - & $\operatorname{sim}$ & $\operatorname{sim}$ \\
\hline Baixo peso ao nascer (\%) & $\begin{array}{l}\text { Média } \\
\text { (DP) }\end{array}$ & $\begin{array}{l}6,71 \\
(1,28)\end{array}$ & $\begin{array}{l}7,23 \\
(1,62)\end{array}$ & $\begin{array}{l}8,47 \\
(1,43)\end{array}$ & 0,0000 & - & $\operatorname{sim}$ & $\operatorname{sim}$ \\
\hline Cobertura do PSF (\%) & $\begin{array}{l}\text { Média } \\
\text { (DP) }\end{array}$ & $\begin{array}{l}32,42 \\
(20,16)\end{array}$ & $\begin{array}{l}31,05 \\
(16,65)\end{array}$ & $\begin{array}{l}25,96 \\
(20,57)\end{array}$ & 0,1997 & - & - & - \\
\hline
\end{tabular}

* Teste de Dunn: diferença significante $(p<0,05) . /{ }^{*}$ Dunn's test: statistically significant difference $(p<0,05)$. 

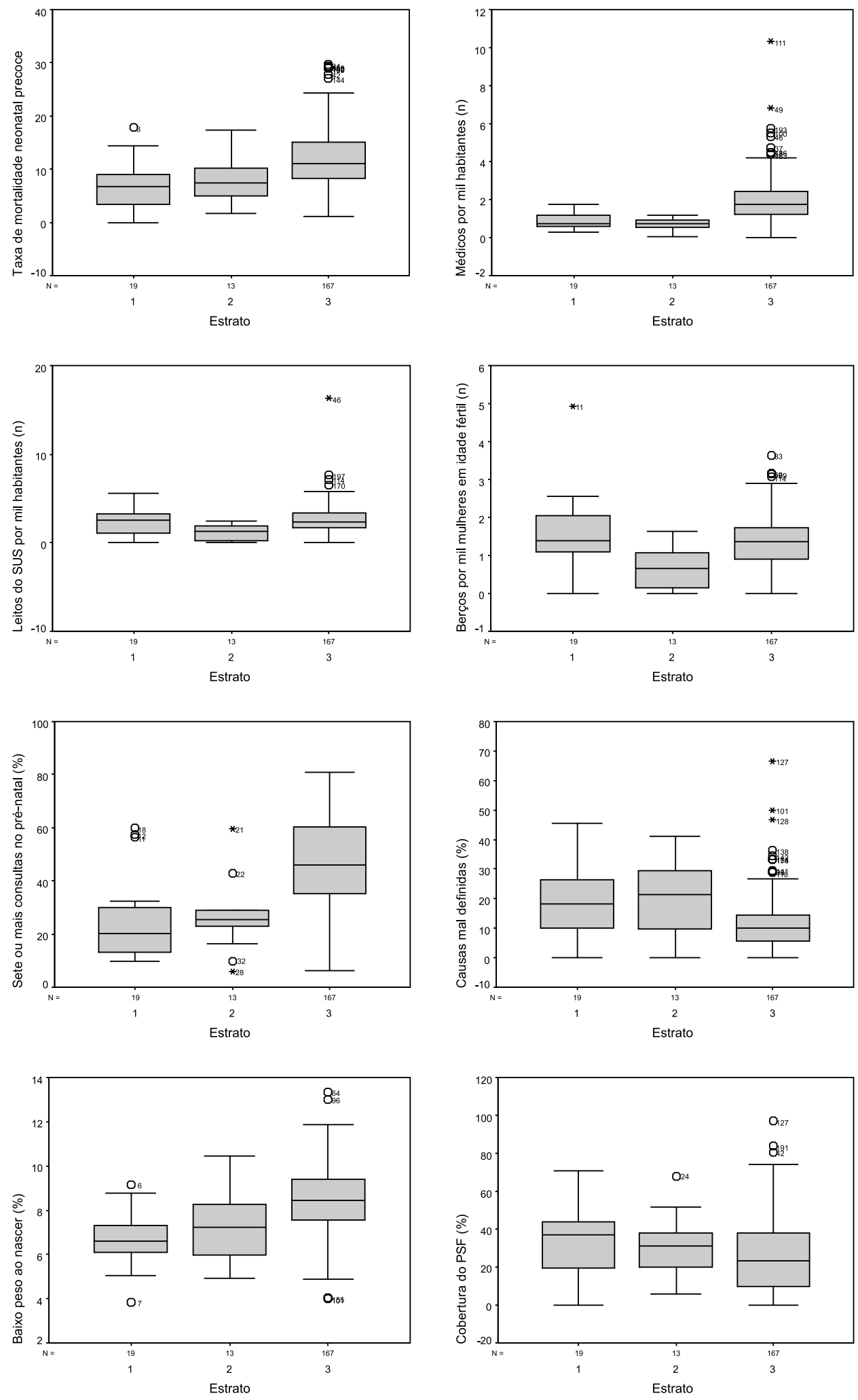

Figura 2 - Distribuição de variáveis assistenciais selecionadas segundo os estratos da árvore de classificação, Minas Gerais, 1999-2001.

Figure 2 - Distribution of selected care variables according to city group strata from the classification tree, Minas Gerais, 1999-2001. 
- Estrato 3: Médicos por habitantes correlacionou-se negativamente com causas mal definidas e cobertura do $P S F$, e positivamente com as demais variáveis. Leitos por habitantes, berços por mulher e sete ou mais consultas no pré-natal correlacionaram-se positivamente. A correlação foi negativa entre causas mal definidas e sete ou mais consultas no pré-natal e baixo peso ao nascer, e positiva com cobertura do PSF (dados não apresentados).

Diante dos resultados obtidos nas análises descritiva e de correlação, considerou-se que a TMNP_SIH poderia apresentar problemas de sub-registro. Buscando dados de melhor qualidade para investigar a associação entre a TMNP_SIH e as variáveis assistenciais, foram selecionados os grupamentos de municípios pertencentes a microrregiões que alcançaram grau de cobertura do SIM maior ou igual a $90 \%$, segundo estudo re- alizado pela Fundação João Pinheiro $(2005)^{\mathrm{b}}$, considerando que é no hospital que se preenchem as Declarações de Óbito (DOs) e as AIHs. Dos 99 grupamentos de municípios com essas características, 98 pertenciam ao Estrato 3 e foram selecionados para a análise. A TMNP_SIH média foi de 13,2 por mil NV. Em relação aos demais grupamentos de municípios, estes 98 grupamentos tinham melhor perfil socioeconômico e melhores resultados para as variáveis sete ou mais consultas no pré-natal (mediana de 53,5\%) e causas mal definidas (mediana de $8,7 \%$ ). Observou-se correlação positiva e significativa da TMNP_SIH somente com berços por mulher, significando que apenas esta, entre as variáveis assistenciais, poderia explicar o comportamento da TMNP_SIH. Médicos por habitantes correlacionou-se positiva e significativamente com as demais variáveis assistenciais, exceto causas mal definidas, quando a correlação foi negativa (resultados não apresentados).

Tabela 3 - Matriz de correlação de Spearman entre a taxa de mortalidade neonatal precoce no $\mathrm{SIH} / \mathrm{SUS}$ e variáveis assistenciais em cada estrato, Minas Gerais, 1999-2001

Table 3 - Spearman correlation matrix in municipal groups by classification tree stratus, Minas Gerais, 1999-2001.

\begin{tabular}{lccc}
\hline Variáveis & \multicolumn{3}{c}{ Estrato } \\
\hline Médicos por habitantes (n) & 1 & 2 & 3 \\
Leitos por habitantes (n) & 0,416 & 0,308 & $0,168^{*}$ \\
Berços por mulher (n) & 0,396 & 0,000 & 0,053 \\
Sete ou mais consultas no pré-natal (\%) & $-0,423$ & 0,286 & 0,128 \\
Causas mal definidas (\%) & 0,037 & $-0,302$ & $-0,013$ \\
Baixo peso ao nascer (\%) & $0,558^{*}$ & $-0,082$ & 0,050 \\
Cobertura do PSF (\%) & $-0,354$ & 0,209 & $-0,120$ \\
\hline
\end{tabular}

*Correlação é significante ao nível de 0,05/Correlation is significant at the 0,05 level (2-tailed)

**Correlação é significante ao nível de 0,01/Correlation is significant at the 0,01 level (2-tailed)

b Fundação João Pinheiro. Centro de Estudos Econômicos e Sociais. Mensuração e acompanhamento do nível da mortalidade infantil de Minas Gerais. - Belo Horizonte, 2005. 99 p.iil. 


\section{Discussão}

Ao contrário do esperado, os Estratos 1 e 2, de grupamentos de hospitais de municípios com pior nível socioeconômico, apresentaram maior probabilidade de a TMNP_SIH ser menor que 8 por mil NV. Ao mesmo tempo, esperava-se que maiores TMNP_SIH ocorressem em municípios com menores valores de médicos por habitantes, leitos por habitantes e berços por mulher. Entretanto, o contrário foi observado em todos os estratos e a correlação, mesmo quando não significativa, foi positiva. Ou seja, a TMNP_SIH era mais baixa onde o número de médicos por habitantes tinha valores mais reduzidos.

Maior proporção de óbitos infantis por causas mal definidas revela possíveis problemas na assistência médica nos Estratos 1 e 2. A pequena proporção de mães que realizaram sete ou mais consultas no pré-natal e de pessoas cadastradas no programa de saúde da família e as elevadas taxas de pobreza e de analfabetismo sugerem maiores problemas de acesso aos serviços de saúde nos Estratos 1 e 2, se comparados ao Estrato 3 (Tabela 2).

Fica então a pergunta: não se verificou correlação significativa entre a TMNP_SIH e as variáveis assistenciais porque ela realmente não existe? Ou seja, os indicadores de condições estruturais não garantem a existência de assistência adequada e, portanto, não garantem menores TMNP_SIH? Ou seria porque os dados são questionáveis? A pior qualidade dos dados poderia explicar a inexistência de correlação significativa ou uma correlação de sentido inesperado (ou positiva ou negativa). Dessa forma, os baixos níveis da TMNP_SIH observados nos grupamentos de municípios localizados nas regiões do norte e nordeste mineiros podem ser indicadores de sub-registro desses óbitos no SIH/SUS.

Com metodologia semelhante à do presente estudo, Schramm \& Szwarcwald $(2000 a)^{1}$ identificaram taxas de mortalidade neonatal precoce muito baixas no $\mathrm{SIH} /$
SUS em 1995 nos Estados de Piauí e Maranhão (2,6 e 3,4 por mil NV, respectivamente), que inferiram ser provavelmente devidas a erro de classificação entre nascido vivo e nascido morto. Por outro lado, em São Paulo a taxa foi de 12,0/1000 NV e, em Minas Gerais, de 10,4/1000 NV, semelhante ao nível verificado neste trabalho. As autoras explicam esses resultados pelos limites impostos, por um lado, pela baixa oferta e pelas dificuldades de acesso aos serviços de saúde nos Estados do Norte e Nordeste do Brasil; por outro, pelas altas taxas de mortalidade neonatal precoce nas regiões mais desenvolvidas, que refletem a qualidade da assistência. No mesmo sentido, Lemos (2004) $)^{19}$, estudando as microrregiões de Minas Gerais, observou uma inesperada alta correlação negativa entre o índice de exclusão social e a proporção de crianças com baixo peso ao nascer e o percentual de gestantes com seis ou mais consultas no pré-natal, mas alta correlação positiva com causas mal definidas de óbitos. No Estado do Paraná, Andrade et al. (2006) ${ }^{20}$ encontraram melhor qualidade das informações de rotina do Ministério da Saúde (MS) sobre nascimentos e óbitos e de cobertura em municípios localizados no cluster de maior nível socioeconômico, e uma taxa de mortalidade neonatal menor no cluster de pior condição de vida, provavelmente devida ao sub-registro de óbitos, classificação errônea de óbitos neonatais como óbitos fetais, problemas no fluxo das informações ou migração de óbitos para áreas de melhores condições de assistência.

As grandes distâncias geográficas, aliadas ao fato de que os caminhos não são asfaltados e que empecilhos ainda maiores ocorrem na época de chuvas, tornam muito desfavorável a realidade das pessoas que vivem nas regiões de Jequitinhonha, Nordeste, Noroeste e Norte de Minas. Podem ter ocorrido óbitos em situações em que as gestantes não conseguiram alcançar o hospital, especialmente as residentes em municípios pobres e de grande extensão rural, e que não possuem hospitais 
conveniados ao SUS, como observado neste estudo no Estrato 2. Por exemplo, em 2001, na macrorregião Nordeste e na microrregião de Almenara, a proporção de partos registrados como domiciliares foi de $7,5 \%$ e de $16,4 \%$, respectivamente, muito superiores à média do Estado (dados não apresentados). Este raciocínio é corroborado pelo estudo realizado por Mello Jorge et al. (1997) ${ }^{21}$ em Londrina, que revela que, entre os motivos apresentados por mães que tiveram partos registrados como domiciliares, estão a falta de meio de locomoção para chegar ao hospital, nascimento durante o trajeto e acesso difícil. Oliveira et al. (2004) $)^{6}$ estudando a probabilidade de internação na rede SUS no Brasil, demonstram a desigualdade no acesso ao serviço. Pacientes residentes em municípios mais pobres, que têm o perfil da clientela dependente do SUS, precisam superar a distância a ser percorrida (e os custos) em busca da assistência hospitalar em outro município. Mesmo distâncias geográficas muito pequenas impõem redução na probabilidade de atendimento em virtude das condições socioeconômicas da população.

A cobertura do PSF não apresentou diferenças entre os estratos, indicando que, mesmo nas áreas com menor nível socioeconômico, esta estratégia de atenção à saúde e de diminuição das desigualdades no acesso e na utilização dos serviços de saúde $^{22}$ não está completamente implantada. Além disso, provavelmente seu impacto seria maior sobre a mortalidade infantil tardia do que sobre a mortalidade neonatal precoce, onde atuaria apenas de forma indireta através da melhoria da saúde materna e do início precoce do cuidado pré-natal ${ }^{23}$.

Neste estudo, verificou-se uma aparente consistência entre os níveis de TMNP_SIH e a proporção de baixo peso ao nascer, ou seja, uma correlação positiva entre essas variáveis nos grupamentos do
Estrato 1. Entretanto, menor proporção de baixo peso ao nascer nos grupamentos de menor nível socioeconômico pode se configurar no denominado paradoxo epidemiológico ${ }^{24}$. É possível que esses resultados se devam à influência da percepção da viabilidade do recém-nascido por parte do profissional de saúde, conduzindo à classificação errônea de crianças nascidas vivas como natimortas, especialmente nas primeiras horas de vida. Por outro lado, a maior probabilidade de intervenções obstétricas em gestantes de maior risco e maior sobrevida dos recém-nascidos em hospitais com melhor qualidade da assistência nas áreas com maior nível socioeconômico ${ }^{24,25}$ poderia explicar a ocorrência de maior proporção de baixo peso ao nascer e maiores TMNP_SIH verificados no Estrato 3. Resultado semelhante foi observado por Leal e Szwarcwald (1997) ${ }^{26}$ no Estado do Rio de Janeiro entre 1979 e 1992, com menores taxas de mortalidade nas primeiras 23 horas de vida ocorrendo nos municípios de pior situação socioeconômica, provavelmente devido ao maior sub-registro de óbitos.

Trabalhos recentes realizados em Minas Gerais apontam inadequação das informações sobre óbitos e nascimentos em várias regiões do Estado com dezessete microrregiões do Nordeste, Norte de Minas, Jequitinhonha, Noroeste e Leste classificadas como tendo informações muito precárias $^{\mathrm{c}, 27}$. Pode ser que alguns problemas que resultem em sub-registro no SIM e no SINASC se repitam em relação ao SIH/ SUS, pois as mesmas instituições e, provavelmente, os mesmos profissionais são responsáveis pelo preenchimento dos formulários que alimentam os três sistemas. Melo et al. (2004) ${ }^{28}$ e Côrtes (2002) ${ }^{29}$ encontraram sub-registro de óbitos por infarto agudo do miocárdio e meningites no SIH/ SUS, quando este foi comparado a outros sistemas de informação de rotina ou ao prontuário. É possível que erros de digitação tenham ocorrido na transcrição de

c Fundação João Pinheiro. Centro de Estudos Econômicos e Sociais. Mensuração e acompanhamento do nível da mortalidade infantil de Minas Gerais. - Belo Horizonte, 2005.99 p.iil. 
dados do prontuário para a $\mathrm{AIH}$ e na codificação dos óbitos, o que pode ser minimizado pelo treinamento continuado e a padronização de critérios de preenchimento dos campos da $\mathrm{AIH}^{28}$.

$\mathrm{Na}$ análise dos resultados deste estudo é necessário considerar algumas possíveis limitações. Como delineamento ecológico, foram utilizadas variáveis assistenciais e socioeconômicas agregadas referentes ao conjunto da população dos municípios, enquanto a variável resposta referiu-se apenas à parcela da população usuária do SUS, correspondente a cerca de $80 \%$ do total de partos ${ }^{30}$. Sabe-se, entretanto, que a cobertura de partos é diferenciada e que, quanto mais baixa a renda, maior a proporção da população usuária do SUS ${ }^{6}$. Desta forma, é provável que nos municípios mais ricos ocorra maior cobertura de partos por convênios médicos. Por outro lado, neste estudo foi justamente nas áreas mais carentes que foi observada maior proporção de partos domiciliares e menores TMNP_SIH. Podemos então considerar que a TMNP_SIH seria também um indicador de oferta de serviços. No caso dos municípios do Estrato 3, além do atendimento SUS proporcionalmente maior para a população pobre, é provável que pacientes de áreas mais carentes migrem em busca de atendimento especializado, contribuindo para aumentar artificialmente o risco de eventos adversos em áreas de maior nível socioeconômico e melhor oferta de serviço médico. Assim, dois mecanismos estariam atuando: primeiramente, é possível que a gestante, residente em municípios limítrofes dos grandes centros urbanos, forneça outro endereço no município do hospital para garantir o atendimento $^{31}$, especialmente se for o caso de uma gravidez de alto risco. Em segundo lugar, o atendimento nos hospitais do SUS pode refletir proporcionalmente mais os bolsões de pobreza dos grandes municípios do Estrato 3, que apresentam maior proporção de domicílios subnormais e maior risco de morte neonatal.

Nas localidades de Minas Gerais com pior qualidade de dados do SINASC e SIM $^{\mathrm{d}, 27}$, o SIH/SUS está captando melhor os eventos, ou seja, foram maiores as razões entre nascimentos e óbitos registrados no SIH/SUS e no SINASC e SIM. O elevado número de nascimentos e de óbitos neonatais precoces registrados no SIH/SUS justifica o estudo dos dados coletados por este sistema e o investimento na qualidade desses dados. Sabe-se que somente o seu uso continuado em estudos epidemiológicos poderá contribuir para a melhoria da qualidade dos registros. O uso do SIH/SUS deve, portanto, ser estimulado.

No presente trabalho alguns cuidados metodológicos foram adotados com o objetivo de evitar taxas muito instáveis associadas ao uso de pequenos números. Foram utilizados três anos e os hospitais foram agrupados buscando-se alcançar pelo menos $250 \mathrm{NV}$ no denominador das TMNP_SIH. Os municípios foram agrupados respeitando-se os limites das microrregiões para levar em conta a lógica de fluxo de pessoas e a disponibilidade de recursos segundo o Plano Diretor de Regionalização da Secretaria de Estado de Saúde de Minas Gerais ${ }^{13}$. Entretanto, o nível de desagregação da unidade de análise, grupamento, possivelmente não foi capaz de detectar diferenças entre os hospitais dentro de um mesmo grupo, os quais podem apresentar comportamentos bem distintos. Quanto aos indicadores selecionados, os valores medianos possivelmente representaram de forma mais adequada os grupamentos de municípios dentro de cada estrato, devido à grande variabilidade dos dados.

As análises aqui realizadas levantaram a hipótese de que as TMNP_SIH eram subregistradas em alguns locais. Desta forma, métodos estatísticos mais elaborados, mesmo os mais robustos, que não levem em

d Fundação João Pinheiro. Centro de Estudos Econômicos e Sociais. Mensuração e acompanhamento do nível da mortalidade infantil de Minas Gerais. - Belo Horizonte, 2005. 99 p.il. 
conta este possível erro podem levar a inferências equivocadas ou de pouca validade $^{12}$. Em relação ao método CART utilizado, a análise descritiva em cada estrato reforçou a sua validade interna. Apesar dos problemas detectados em relação aos níveis das TMNP_SIH em Minas Gerais, o método foi capaz de estratificar os grupamentos de municípios, discriminando as desigualdades socioeconômicas de modo satisfatório. Dessa forma, observa-se que existem três "Minas": uma mais rica e populosa com mais recursos em saúde, o Estrato 3; outra mais pobre, porém com alguma assistência à saúde; e a terceira mais pobre ainda e com menor assistência, especificamente com relação ao atendimento ao pré-natal, ao parto e ao recém-nascido. A maior probabilidade de TMNP_SIH mais baixas nos Estratos 1 e 2 onde a situação socioeconômica é mais precária, possivelmente se deve ao sub-registro dos óbitos neonatais precoces nesses locais. Tal observação nos leva a considerar que a desigualdade social e econômica se apresen- ta também na qualidade dos registros das taxas de mortalidade neonatal precoce hospitalar através de diferentes mecanismos. Para avaliar melhor estes mecanismos são necessários estudos mais aprofundados e mais próximos do local de ocorrência dos eventos e dos processos de trabalho.

\section{Agradecimentos}

Agradecemos muito especialmente a Daisy Abreu (NESCON/UFMG) pelo apoio e sugestões sempre oportunos, a Eliane Drumond (SMS/Belo Horizonte) e Maria do Carmo Raushi (SES/Minas Gerais) pelas preciosas sugestões e ponderações, e a Luís Monteiro (FM/UFMG) pela colaboração com os bancos de dados. Este trabalho foi parcialmente financiado por FAPEMIG (Processo EDT 1770/03) e CNPq (Processo no 403707/04-8). RHL tem sua pesquisa parcialmente financiada pelo CNPq (Processos no 3004505/2006-4 e 472877/2006-2 e 472066/2004-8). DC recebeu bolsa de mestrado da CAPES.

\section{Referências}

1. Schramm JMA, Szwarcwald CL. Diferenciais nas taxas de mortalidade neonatal e natimortalidade hospitalares no Brasil: um estudo com base no Sistema de Informações Hospitalares do Sistema Único de Saúde (SIH/SUS). Cad Saúde Pública 2000a; 16(4): 1031-40.

2. Szwarcwald CL, Leal MC, Castilho EA, Andrade CLT. Mortalidade infantil e no Brasil: Belíndia ou Bulgária? Cad Saúde Pública 1997; 13(3): 503-16.

3. Andrade CLT, Swarzwald CL, Gama SGN, Leal MC. Desigualdades socioeconômicas do baixo peso ao nascer e da mortalidade perinatal nos Municípios do Rio de Janeiro, 2001. Cad Saúde Pública 2004; 20(S1): 44-51.

4. Lawn JE, Cousens S, Zupan J for the Lancet Neonatal Survival Steering Team. 4 million neonatal deaths: When? Where? Why? Publish online March 3, 2005. Disponível em http://image.thelancet.com/extras/ 05art1073web.pdf. (Acessado em 18 de março de 2005)

5. Dever GEAlan. A epidemiologia na administração dos serviços de saúde. São Paulo: Pioneira; 1988.

6. Oliveira EXG, Travassos C, Carvalho MS. Acesso à internação hospitalar nos municípios brasileiros em 2000: territórios do Sistema Único de Saúde. Cad Saúde Pública 2004; 20(S2): 298-309.
7. Lansky S, França E, Leal MC. Mortalidade infantil e evitabilidade: revisão da literatura. Rev Saúde Pública 2002; 36(6): 759-72.

8. Ministério da Saúde. Informações de saúde [on-line]. Disponível em http://www.datasus.gov.br. (Acessado durante o ano de 2005, para informações de 1995 a 2002. .)

9. Leal MC, Viacava F. Maternidades do Brasil. RADIS 2002; 2: 8-26.

10. Machado ENM, Alfradique ME, Monteiro LP. Caracterização da rede hospitalar do Sistema Único de Saúde em Minas Gerais. Fundação João Pinheiro: Belo Horizonte; 2003.

11. Brasil. Ministério da Saúde. Secretaria de Vigilância à Saúde. Departamento de Análise de Situação em Saúde. Saúde Brasil - 2004: uma análise da desigualdade em saúde. Brasília: Ministério da Saúde; 2006.

12. Schramm JMA, Szwarcwald CL. Sistema Hospitalar como fonte de informações para estimar a mortalidade neonatal e a natimortalidade. Rev Saúde Pública 2000b; 34(3): 272-9. 
13. Minas Gerais. Secretaria de Estado de Saúde. Superintendência de Planejamento e Coordenação. Plano Diretor de Regionalização de Minas Gerais - 2001/2004. Belo Horizonte (MG): Coopmed; 2002.

14. Programa das Nações Unidas para o Desenvolvimento/ Instituto de Pesquisa Econômica Aplicada/Fundação João Pinheiro. Atlas do Desenvolvimento Humano no Brasil. [CD-ROM]. Belo Horizonte: Fundação João Pinheiro; 2003.

15. Breiman L, Friedman JH, Olshen RA, Stone CJ. Classification and regression trees. New York: Chapman \& Hall; 1984

16. Ferreira CA. Comparação da capacidade preditiva da regressão logística, CART e redes neurais [dissertação de mestrado]. Belo Horizonte: Departamento de Estatística da UFMG; 1999.

17. Martinez EZ, Louzada-Neto F, Pereira BB. A curva ROC para testes diagnósticos. Cad Saúde Coletiva 2003; 11(1): 7-31

18. Statistical Package for the Social Sciences - SPSS Answer Tree. [computer program]. Versão 3.0. Chicago, Illinois: SPSS Inc.; 2001.

19. Lemos MMV. Atlas de Minas Gerais: a saúde e o social sob a luz do plano diretor de regionalização [dissertação de Mestrado]. Uberlândia: Programa de Pós-Graduação em Geografia da UFU; 2004.

20. Andrade SM, Soares DA, Matsuo T, Souza RKT, Mathias TAF, Iwakura MLH, et al.. Condições de vida e mortalidade infantil no Paraná, Brasil, 1997/2001. Cad Saúde Pública 2006; 22(1): 181-9.

21. Mello-Jorge MHP, Gotlieb SLD, Andrade SM. Análise dos registros de nascimentos vivos em localidade urbana no Sul do Brasil. Rev Saúde Pública 1997; 31(1): 78-89.

22. Goldbaum M, Gianini RJ, Novaes HMD, César CLG Utilização de serviços de saúde em áreas cobertas pelo programa saúde da família (Qualis) no Município de São Paulo. Rev Saúde Pública 2005; 39(1): 90-9.
23. Macinko J, Guanais FC, Souza MFM. Evaluation of the impact of the Family Health Program on infant mortality in Brazil, 1990-2002. J Epidemiol Community Health 2006; 60: 13-19.

24. Silva AAM, Bettiol H, Barbieri MA, Pereira MM, Brito LGO, Ribeiro VS et. al.. Why are low birthweight rates in Brazil higher in richer than in poorer municipalities? Exploring the epidemiological paradox of low birthweight . Paediatric And Perinatal Epidemiology 2005; 19: 43-9.

25. Joseph KS, Demissie K, Kramer MS. Obstetric intervention, stillbirth and preterm birth. Semin Perinatol 2002; 26(4): 250-9.

26. Leal MC, Szwarcwald CL. Características da mortalidade neonatal no Estado do Rio de Janeiro na década de 80: uma visão espaço-temporal. Rev Saúde Pública 1997; 31(5): 457-65.

27. França E, Abreu D, Campos D, Rausch MC. Avaliação da qualidade da informação sobre mortalidade infantil em Minas Gerais, em 2000-2002: utilização de uma metodologia simplificada. Rev Méd Minas Gerais 2006; 16(S2): 28-35.

28. Melo ECP, Travassos C, Carvalho MS. Qualidade dos dados sobre óbitos por infarto agudo do miocárdio, Rio de Janeiro. Rev Saúde Pública 2004; 38(3): 385-91.

29. Côrtes MCJW. Vigilância das meningites na Região Metropolitana de Belo Horizonte, MG, 1999: o uso dos sistemas de informação em saúde e o método da capturarecaptura na estimação da incidência e da subnotificação [tese de doutorado]. Belo Horizonte: Departamento de Medicina Tropical, Faculdade de Medicina da UFMG; 2002.

30. Moreira ML. Cobertura e utilização de serviços de saúde complementar no Estado de São Paulo [dissertação de mestrado]. Rio de Janeiro: Fiocruz, Escola Nacional de Saúde Pública; 2004

31. Souza RKT, Gotlieb SLD. Sub-registro de nascimentos vivos hospitalares em área urbana da região sul do Brasil, 1989. Rev Saúde Pública 1993; 27(3): 177-84.

Recebido em: 25/08/06 Versão final reapresentada em: 09/04/07 Aprovado em: 09/05/07 